\title{
Advanced Nursing Education: Critical Factors that Influence Diploma and Associate Degree Nurses to Advance
}

\author{
Rose Lavine McGhie-Anderson PhD, ARNP, MSc, CEN, RN*
}

\begin{abstract}
${ }^{1}$ Lecturer of Maternal and Newborn Health Nursing, Faculty of Nursing, Menoufiya University, Egypt And Ass. Prof in College of Applied Medical Sciences, Shqura University, KSA.

${ }^{2}$ Lecturer of Maternal and Newborn Health Nursing, Faculty of Nursing, Menoufiya University, Egypt.
\end{abstract}

"Correspondence:

Rose Lavine McGhie-Anderson, 2074 SW 173 rd Avenue Miramar, Florida, USA, Tel: 9546638528; E-mail: havergal321@gmail.com.

Received: 19 April 2018; Accepted: 14 May 2018

Citation: Rose Lavine McGhie-Anderson. Advanced Nursing Education: Critical Factors that Influence Diploma and Associate Degree Nurses to Advance. Nur Primary Care. 2018; 2(3): 1-6.

\section{ABSTRACT}

Aim: To gain an understanding of the social processes associated with the decision of diploma and associate degree nurses to advance academically.

Background: Advanced nursing education needs to be pursued along the continuum of the nursing career path. This education process is indispensable to the role of nurses as educator, manager, nurse leader, and researcher who will effect policy changes, assume leadership roles as revolutionary thinkers, and to implement paradigmatic shifts.

Method: Data were collected from two groups of participants using a face-to-face semi-structured interview. Group one was diploma and associate degree nurses. Group two was baccalaureate, masters, and doctoral degree nurses who have progressed academically.

Results: Emerging from the thick, rich data were core categories of: rewarding, motivating, and supporting as critical factors that influence professional advancement.

Conclusions: This qualitative study elucidated that professional advancement was the social process that grounds. Emergent theory was, The Theory of Professional Advancement.

Keywords

BSN Perception and Education, Advanced Nursing Education, and Continuing Nursing Education

\section{Introduction}

Advanced nursing education is used synonymously with the terms academic progression or professional progression. Academic progression refers to articulation models that promote lifelong learning through the addition of academic credentials [1]. It is therefore a movement of advancement by degrees [2,3]. Having qualified faculty and advanced practice nurses to manage new and emerging environments of care in various settings will be unattainable if approximately $60 \%$ of new nurses are associate degree graduates [1]. Worldwide, nurses are called to work in an environment where there are increasing health care reforms, complicated patient care needs, technological advancements, and improved evidence-based practices. These ongoing changes call for nurses who are competent in leadership, health policy, system improvement, research, evidence-based practice, and collaboration. This is essential in facilitating the delivery of highquality care [1].

Internationally, there are over 14,465,439 nurses. Nationally, there are over 3 million nurses of whom 2.7 million are employed [4]. In the United States, the nursing profession is considered the largest segment of the health care industry with nurses accounting for $55 \%$ of the workforce [5]. Of this workforce, only $50 \%$ hold a baccalaureate degree or higher [3]. The IOM recommends that by $2020,80 \%$ or more of the nursing population should be baccalaureate educated [3]. This indicates that 465,000 diploma and associate degree nurses are to return to school by 2020 [4]. Studies such as Effects of hospital care environment on patient mortality and nurse outcomes Aiken, Sean, Sloane, Lake, and Cheney [6], have shown that the nurse who is academically 
advanced improves patient outcomes. It was revealed that surgical mortality rates exceed $60 \%$ in hospitals with lower educational level nurses, whereas hospitals with higher educated nurses have better patient outcomes [6].

\section{Literature Review}

A review of relevant literature across disciplines was conducted to explore the phenomenon of advanced nursing education: Critical factors that influence diploma and associate degree nurses to advance. This literature review was divided by topics into the major theoretical and research literature addressing what critical factors influence professional advancement for the diploma and associate degree nurses. These topics include: (a) the historical context of advance nursing education, highlighting the trend and development in nursing over the centuries and the direction it needs to take for the future; (b) motivations to advance; and (c) perceptions of advanced education and (4) benefits of advanced nursing education. A synthesis of the literature was provided to explicate what is known and unknown about the factors that influence nurses to advance academically. In exploring the literature, it has been revealed that not many researches were done over the past decade addressing academic advancement [7]. Also in order to increase the percentage of nurses to at least the BSN degree level by 2020, more than 465,000 diploma and ADN nurses must advance academically [8].

Historically, many hospital-based nursing programs collaborated with colleges and universities to meet the educational needs of diploma nurses to transition to associate and baccalaureate degrees. The associate nursing degree program began in the early post World War II and following the Ginzberg Report in 1949, which recommended a two -year nursing program to meet the existing nursing shortage. In 1965, the American Nurses Association (ANA) published a position paper, which recommended that the minimum preparation of the professional nurse be a baccalaureate degree [9]. Research has shown that the baccalaureate educated nurse helps to increase patient outcomes [6]. Magnet hospitals also employ a higher percentage of baccalaureate nurses, and most hospitals prefer a BSN prepared nurse.

Literatures addressing motivations to advance indicate that, countries such as Canada, Sweden, Portugal, Brazil, Greece, Iceland, and the Philippines, all require a 4-year undergraduate degree to practice as a registered nurse [10]. However, there are other countries including the United States where a majority of their nurses have only a diploma or an associate's degree (National Statistics for Registered Nurses [11]. Warren and Mills conducted a quantitative study exploring what motivates nurses to advance professionally. The findings revealed that approximately $19 \%$ of nurses planned to enroll in a nursing degree program if they are offered the right incentives such as career satisfaction, professional commitment, and if having a BSN degree offers opportunities and incentives from their employer. Aiken et al. [6], conducted a retrospective observational study that reviewed 26,516 professionals at the bedside from 2009-2010. The aim of this study was to assess if there were differences in patient to nurse ratios and nurses educational qualifications in nine of the 12 RN4CAST countries that had similar patient discharge data and variations in mortality rates after a common surgical procedures [6]. The implications for this study are that the reduction of nursing staff in hospitals may adversely affect patient outcomes, and BSN degree educated nurses significantly reduce preventable hospital deaths [6].

Osterman et al. [7] explored nurses' perceptions about returning to school for a baccalaureate degree. A qualitative approach was used to understand the meanings and the interpretations of nurses who pursued a BSN degree. The participants revealed that their perceptions of advanced education may be seen in the meaning for them on entering the program and meaning on preparing to graduate or upon graduation, the scope of the participants' knowledge on professionalism, learning to reframe thought processes, seeing the big picture, and the participants' approach to practice [7].

Benefits on academic progression was elucidated in studies such as a qualitative, case study research conducted in Australia to investigate how existing registered midwives are challenged by current statutory requirements, as it relates to continued professional development. The study indicated that health professionals have legal, regulatory, and ethical obligations to remain up-to-date and informed by advancing professionally. The commitment to advance is attributed to motivation as the core theme. "Motivated individuals are commonly attracted to participate in research studies". Eley, Francis, and Hegney also conducted a study on career progression. A quantitative cross-sectional cohort design with mailed survey was used to measure the extent of the relationship between opportunity for career progression in nursing with turnover and retention. The study revealed that the lack of career progression among nurses remains a major concern for patients and families, nurses, and the nursing profession.

\section{Method}

Grounded theory is a qualitative research process used to describe data that are systematically collected and analyzed. The researcher begins with no preconceived conclusions of a theory; instead, the theory emerges from data to provide insight, to enhance understanding, and to provide the interpretations and meanings that may guide action. In a grounded theory methodological approach, there is an existing relationship between data collection, analysis, and the theory that will emerge from the data. A theory that is derived from data is the reality of the participants' social interaction and experiences and is constructed from data rather than discovery. According to Strauss and Corbin [12], "theorizing is the act of constructing" [12]. The researcher asks questions that will stimulate the thoughts of the participants and extract a creative, cohesive, and organized schema from the raw data to which the researcher assigns categories. In order to implement data collection and categorization, following Barry University's IRB approval, the researcher purposefully selected participants from hospitals and colleges of nursing in South Florida who would provide insight to the phenomenon of interest. The sample consisted of 22 participants; there were 15 diploma and associate degree nurses and seven BSN, MSN, and doctoral educated focus 
group members. The data collected were from interviews and observations for the primary purpose of discovering concepts and relationships. This data collection process was implemented in a systematic back-and-forth pattern, constantly comparing what is being collected and examining the dimensions of what is already collected to get a fresh perspective. The data collection and analysis occurred simultaneously. Memo notes are a part of the analytical process and are made by the researcher to record, clarify, and expand the theory as it develops [12]. Memoing can be an inductive as well as a deductive process. It is inductive when the data is being conceptualized and deductive when the researcher assesses the relationship of conceptual labels, categories, and subcategories [12].

Participants who made contact with the researcher indicating willingness to participate in the study were assessed to determine if they met the criteria for participation. Prior to each interview, the participants were informed of the purpose of the study, their rights to participate or to withdraw at any time during the research process without fear of repercussion. They were also allowed time to sign their consent forms and complete their demographic questionnaire and were given a $\$ 10.00$ American Express gift card as a token of appreciation for their participation in the study. Each participant was allowed to use a pseudonym instead of their real names. The interviews were conducted in a quiet environment for recording on a tape recorder in individual study rooms at community libraries; this provides neutrality and privacy. Each session lasted approximately 45-60 minutes. Fifteen individual participants were interviewed. Three were interviewed via Skype, and 12 were interviewed face to face. Consents were obtained for the skyped interviewees via DocuSign. The principal challenge encountered with Skype was the loss of connectivity due to a thunderstorm; however, reconnection went smoothly. The main challenges of the face-to-face interview participants were the time and availability of individuals; this was overcome by rescheduling to more convenient times.

The process of data collection used was one that was purported by Strauss and Corbin [12], which allows a systematic process to collect, analyze, and organize data. The researcher wrote field notes to stay in tune with feelings about what was happening with the data and documented observations and how the researcher's perceptions may impact sensitivity to what was relevant in each encounter with the participants. Sensitivity allowed the researcher to be open minded and to avoid undue influence by preconceived beliefs, knowledge, and experience about the phenomenon of interest [13]. Initially, raw data were analyzed using the line-by-line technique to break down the data and for the researcher to become familiar with concepts and categories being identified. Coding was also done at times by sentence and at times by paragraph as the researcher became familiarized with the data. In this open coding phase, the concepts were related directly to the meaning of the data collected [12]. The researcher implemented a constructivist approach to illuminate the paradigm of the nurses' views about their worlds and the impact of their social environment on their decision making towards advanced education [14].

Nur Primary Care, 2018
Axial coding is a technique used to group similar concepts from the data received from the research participants that are similar concepts and placing them into categories through an abstract ordering process. To achieve this, the researcher implemented the process of the constant comparative analysis to verify the data, to obtain fresh perspectives of the information already retrieved, and to guide the researcher as to what further information was needed to be collected. The researcher continued to sort the data through a selective coding process with the intent to derive an overarching category that links all the other categories. Selective coding occurs in the latter stages of the research process. It is the process used by the researcher to identify a core category that pulls all other categories together. The central category represents the main phenomenon of the study.

The process of open, axial, and selective coding was completed in a cyclical manner to derive data saturation. This was done in order to confirm saturation, increase trustworthiness, and ensure that the selection of the representation of diploma and associate degree nurses represented the whole. The data collection process delineated a predetermined number of participants for this study. Twenty-two participants were purposefully selected for this study. Saturation was reached after the thirteenth individual participant was interviewed and an additional two participants were interviewed to confirm saturation. Hence, the total number of participants for the individual interview was fifteen. The Atlas. ti qualitative data analysis software was used by the researcher to manage and organize the dense data to ensure that the data was parsimonious by conceptualizing the categories obtained from the tedious process of open, axial, and selective coding [12]. See results of this inquiry in Table 1.

The phenomenon of interest was the core problem that shaped the data derived from the participants in this study. This allowed the researcher to consider the interactions and the relationships formulated by participants, their descriptions of shared experiences, interpretations, attitudes, and the decisions for diploma and associate degree nurses to advance academically. The analytical processes that stood out in categorizing and formulating concepts involves the process of asking questions to uncover the deep meanings of what, who, where, when, how, and with what consequences and making constant comparison of data to excavate the real meaning behind each story.

The process of deduction was repetitive and continuous to complete, refine, and review all emerging categories and concepts until all possible domains were exhausted. The thick, rich, data emerged into concepts as directed by the following research questions: 1. What are the critical factors that influence the diploma and associate degree nurses to advance their nursing education? 2. How do diploma and associate degree nurses perceive motivations to advance their nursing education? 3. What factors would motivate nurses to advance academically in their nursing career? [14].

\section{Findings}

The purpose of this study was to unearth the critical factors 
that influence diploma and associate degree nurses to advance academically. The study aimed to gain an understanding of the social process associated with the decisions of diploma and associate degree nurses to advance their nursing education. The researcher was able to interpret the circumstances, intentions, and strategies and verify the meanings of the participants' shared experiences from the descriptions of the nurses' social interactions within their environment. The main categories that emerged delineated the social processes that influence diploma and associate degree nurses to advance academically. These include rewarding, motivating, and supporting as the main categories and role differentiation, passion and positive work environment as subcategories. The subcategories; role differentiation and passion were pulled into motivating as a core category while the main category supporting pulled the subcategory positive work environment. These categories emerged as an indication that these are the critical factors that would influence and ground diploma and associate degree nurses in their decision making towards academic advancement. The definitions assigned to these descriptors were expressed by the participants and is supported by Hertzberg motivation-hygiene theory (1968).

\section{Rewarding}

Rewarding is the sum total of all benefits provided to an employee from their employer. It is seen as all monetary and non-monetary, and psychological payments provided by an organization in return for employee contribution. Rewarding encapsulates every aspect of work that is considered valuable to the employee and includes healthy work environment, employer support, learning opportunities, career development, and benefit packages associated with salary.

Rewarding was found to be a main category occurring frequently in the data collected from the research participants. It was reemphasized as one of the factors that would influence diploma and associate degree nurses to advance academically. It emerged from the data repeatedly, more than the other main categories. Rewarding is categorized as tangible and intangible and is described by the participants as consistent, creative ways of recognition in order to motivate diploma and associate degree nurses towards academic progression. Tangible rewards are concrete, measurable, and observable. Examples of these that the nurses highlighted were: job promotion, salary increase/bonuses, plaques, gift cards, dinners, increased leadership roles and active participation in the decision-making process for patient care. According to Viaflar, rewarding would help to promote advancement within the nursing profession, increase the scope of practice, and increase the opportunity to pursue higher degrees; in addition, she commented it would help to reduce the nursing shortage. According to Conover and Richards [15], numerous states are looking for less costly ways to alleviate the nursing shortage. Nurses such as ARNPs in North Carolina, who practice at their full scope, will bring significant benefit to the state [15]. Zully commented that intangible rewarding should be attractive and motivating. Examples of intangible rewarding that were highlighted by the nurse participants included the following: greater ability to stay current with nursing education and advancement in technology; a sense of fulfillment with their academic achievement; increased knowledge that will enhance safe, competent patient care; an increase in leadership opportunities; and experience in the decision making process in patient care delivery.

\section{Motivating}

Motivating is the drive for action that results in rewarding. It therefore targets the cognitions and behaviors that are directed for action. This action is enhanced when rewards are attributed to performance [16]. Motivating was identified as the second most dominant category that would be the driving force to influence diploma and ADN to advance academically; the study's participants explained motivating to be impactful both intrinsically and extrinsically. As one of the main categories, motivating pulls with it the sub-categories of role differentiation and passion. Virm highlighted that if the organization motivated her, she would become passionate about her academic advancement. Yagle stated, "I am not passionate about nursing; it is my second career. I just needed the money to take care of my family. At the end of each work day, I just want to get home."

\section{Supporting}

A supporting work environment involves all aspects that act and react on the body and mind of an employee. From an organizational psychological perspective, it is the physical, mental, and social environments where employees are working together, and their work is analyzed for increased productivity [17]. A supporting environment was interpreted as the culture of an organization that hinges strongly on professional standards and the subsequent impact on the employees. As a main category, it pulls with it the subcategory positive work environment.

Llaney felt that a positive work environment is one that promotes civility and equal opportunity and one that operates an engaging education department would be beneficial in encouraging nursing advancement. Environmental culture as a major category was seen by the study participants Zully and Yerrkar as necessary in the role of helping to influence advanced nursing education. Supporting group members confirmed that the perceptions of the diploma and ADN participants regarding the factors that hinder advanced nursing education mirrors some of the views they were discussing and also added further comments.

The diagram in figure 2 is a visual representation of the conceptual relationship that developed among the main categories of rewarding, motivating, and supporting, as well as the subcategories of role differentiation, passion, and a positive work environment. The reason for this model is to make visible the categories that emerged from the data and to make known the social processes, the limitations, and the relevance to the study. Depicted in the diagram are the thought processes of the diploma and ADN nurses as interpreted by the researcher to help visualize the interconnections among categories. Rewarding is represented as the blue arrow; this may be tangible and intangible. In this model, the sides of the arrow that is used to illustrate this main category that represents 
the interrelationship, interaction, reflexivity, and intercreativity of the participants in their social environment. A rewarding system is regarded as important in an organization to channel the employees' rewards. It has a positive impact on motivating and is to be fair, equitable, and consistent among employees. A rewarding work environment is one that is motivating for individuals and supporting towards desired outcomes and may influence behaviors towards a desired direction [18]. Central within this model are the arrowheads representing the interconnection of the segments of the diagram and occurring within this dynamic central point is meaning generation and transformational experience. This occurrence and experience leads to action, interaction, and consequences as a direct result of the decisions made by the study participants towards academic progression.

In figure 2, the green arrow illustrates motivating, and the sides of this arrow represent communication, sharing of knowledge, mutual benefit, and interconnectivity for all participants within that environment. Motivating is described as a psychological process that will give purpose and direction for desired behaviors and are intrinsic as well as extrinsic [19]. According to Baron [20], individuals may be influenced by motivating in their decisionmaking to carry out a task and their performance may be energized with passion whenever it is followed by rewarding. The red arrow represents supporting, a positive work environment, and culture of the organization where the participants are employed. As a factor that influences advanced education, the interconnection shown by the arrowhead contributes to the intercultural connection between nurses and each employer's cultural experience and what they bring into the environment. An organization's mission, values, philosophy, and norms also shape, support, and create a dynamic intercultural experience and generate meanings that influence academic progression. The diagram expressing the relationship between the categories depicts the integration of the factors that are postulated as the critical components that will influence nurses to progress academically, and the theory that may emerge to support academic advancement [14].

\section{Formulated Theory}

A conceptual framework is described by Miles and Huberman [21], as a written or visual product that explains graphically or narratively the core things to be studied, including key factors, concepts or variables, and the relationship(s) among them [21]. The theory of professional advancement emerged from the data as the basic social process that expounds the critical factors that influence diploma and associates degree nurses to advance academically. It emerged from the categories interpreted and constructed by the researcher as rewarding, motivating, and supporting among diploma and associate degree nurses to advance academically. This is interpreted by the researcher as the theory of what is out there in the communities of these nurses and is a problematic factor that involves consequences that impact academic advancement. Studies have shown that the history of an organization, in which there is an environment that is rewarding and motivating towards nurses' academic pursuits will promote the decision-making towards professional advancement. It is believed that supporting in organizational structure, values, and mission will impact the patterns of interaction, behavior, and the commitment of employees [22]. Organizational cultural environment consists of the collective thinking, habits, attitudes, feelings, practices, and behavior patterns of the employees [23]. Research indicates that the culture that exists in a health care environment is impactful on the nurses' attitudes, knowledge, practices, and motivations to advance academically [24]. These interactions and relationships are interpreted by the participants who attribute meaning to their reality [13].

The degree of a supporting, positive work environment reveals the impact this has on employees and is substantiated by the level of effect on health care organizations and the circular influence on diploma and associate degree nurses. The study participants' interpretation was evidenced by the number of nurses who are still unprepared to advance professionally. The categories that emerged from the dense data that produce salience and a pattern were interpreted by the researcher through a process of repeatedly examining the dimensions and properties of the emerging thoughts and feelings of the participants, reviewing the context of the storyline and the conditions that existed during the interactive relationships of the diploma and associate degree nurses, their actions, and their resulting consequences [25]. There is no existing specificity for the creativity in the art and science of grounded theory research [12]. However, throughout the interplay between the data, the researcher noted the persistent reoccurrence of the participants' discussion of the factors that would influence them to advance academically.

\section{Limitations}

There are limitations regarding the groups of diploma and associate degree nurses that may not be represented across regions and cultures. Only diploma and associate degree nurses were used as individual participants. Participants may have provided information that they thought the researcher wants to hear. Geographical location was a factor for meeting sessions with participants [14].

\section{Recommendations}

The theory of professional advancement that emerged from this study provides a model of why diploma and associate degree nurses would advance academically. This theory has shed light on a social relationship that might have gone unnoticed or misunderstood. However, this is not an end in and of itself but an avenue for further research investigations addressing advanced nursing education among diploma and associate degree nurses and for strategy development for advancement by degrees. The demand for nurses to advance academically is increasing as technology increased, as people can present themselves anywhere health care is offered, as employers increasingly hire nurses who will provide quality and safe care. The emergent categories of: rewarding, motivating, and supporting undergird the theory of professional advancement as the middle range study that was derived from the data provided to the researcher hat is substantive and provisionally verified. As a result, each of the emergent factors may be encouraged, promoted, and 
implemented in the work environment and evaluated for increase in the number of academic advancement among employees. This will also allow for further research regarding the degree of impact on advanced education among nurses. This study may be replicated nationally as well as internationally [14].

\section{Conclusion}

It became apparent throughout the data analysis process that diploma and associate degree nurses who are not advancing academically are experiencing various factors that are impacting their decision-making in influential dimensions. This impact subsequently leads to the nurses' inevitable choices, their decisionmaking towards progressing academically, the resulting outcomes of their choices, and their related consequences. Strategies to promote a positive impact would be directly linked to the factors the nurses interpreted in their environment. Analysis of the data revealed many interrelated factors that impacted the nurses decision-making process towards academic advancement such as having a passion for nursing itself, various supporting factors such as family, community, and work-related support, also were being able to distinguish a clearly defined role for diploma, ADN, and BSN educated nurses. These along with other subcategories are interwoven in the main categories. Three main categories were explicated from the social processes that the participants highlighted repeatedly to formulate the core category; these are: rewarding, which may influence positive behaviors and may be tangible or intangible; motivating, which is a driving force that stimulates action may be derived intrinsically or extrinsically; and supporting, which was alluded to a positive demonstration in organizational culture of encouragement from the employer to strengthen and help to mobilize employees in desired direction. Hence, the categories undergird and ground the theory of professional advancement by the categories of: rewarding, motivating, and supporting among diploma and associate degree nurses to advance academically and describes a social process within the environment of diploma and associate degree nurses that impacts academic progression and led to the emergence of the theory of professional advancement [14].

\section{References}

1. NLN Vision Series Transforming nursing education: Leading the call to reform. Academic Progression in Nursing Education: A living document from the National League for Nursing. 2011.

2. Birks M, Chapman Y, Francis K. Becoming professional by degrees A grounded theory study of nurses in Malaysian Borneo. Singapore Nursing Journal. 2010; 37: 31-42.

3. Institute of Medicine. The future of nursing: Focus on education. 2011.

4. Health Resources and Services Administration. The registered nurse population: Findings from the 2008 National Sample Survey of Registered Nurses. Washington, DC: US Department of Health and Human Services. 2010.

5. Sigma Theta Tau International. Technological informatics guiding education reform. 2006.
6. Aiken L, Sean PS, Sloane D, et al. Effects of hospital care environment on patient mortality and nurse outcomes. National Institute of Health. 2008; 38: 223-229.

7. Osterman PL, Asselin ME, Cullen HA. Returning for a baccalaureate: A descriptive exploratory study of nurse's perceptions. Journal for Nurses in Staff Development. 2009; 25: 109-117.

8. Bureau of Labor \& Statistics Labor force statistics from the current population survey. 2010.

9. American Nurses Association. Nursing's social policy statement: The essence of the profession. books.google.com. 2010.

10. AACN. (2013a). Articulation agreements among nursing education programs. Retrieved from www.aacn.nche.edu... fact sheets American Organization of Nurse Executives (2006). Educating nurses: A call for radical transformation.

11. National Sample Survey of Registered Nurses. The registered nurse population - Bureau of Health Professions. 2008.

12. Strauss AL, Corbin JM. Basics of qualitative research Grounded theory techniques and procedures for developing grounded theory (2nd ed). Thousand Oaks, CA: Sage. 1998.

13. Charmaz K. Constructing grounded theory A practical guide through qualitative analysis. Thousand Oaks, CA: Sage. 2006.

14. McGhie-Anderson. Advanced Nursing Education: Critical factors that influence diploma and associate degree nurses to advance. Unpublished Dissertation. 2016.

15. Conover C, Richards R. Economic benefits of less restrictive regulation of advanced practice nurses in North Carolina. Nursing Outlook. 2015; 63: 585-592.

16. Miller EM, Shankar MU, Knutson B, et al. Dissociating motivation from reward in human striatal activity. Journal of Cognitive Neuroscience. 2014; 26: 1075-1084.

17. Jain R, Kaur S. Impact of work environment on job satisfaction. International Journal of Scientific and Research Publications. 2014; 1 .

18. Hafiza NS, Shah SS, Jamsheed H, et al. Relationship between rewards and employee's motivation in the non-profit organizations of Pakistan. Business Intelligence Journal. 2011; 4: 327-334.

19. Rahim MA, Wan Daud WN. A proposed conceptual framework for rewards and motivation among administrators of higher educational provider in Malaysia. International Journal of Business and Commerce. 2012; 1: 67-78.

20. Baron RA, Allyn, Bacon. Behavior in organizations. 1983.

21. Miles MB, Huberman AM. Qualitative data analysis: An expanded sourcebook, 2nd Edition. Thousand Oaks, California, Sage Publication. 1994.

22. Greenspan CMN. Culture clashes. Executive Excellence. 1999; 16: 12 .

23. Mohelska H, Sokolova M. Organizational culture and leadershipJoint vessels. Science Direct. 2015; 171: 1011-1016.

24. Fedorowsky R, Peles-Bortz A, Masarwa S, et al. CarbapenemResistant Enterobacteriaceae carriers in acute care hospitals and post-acute-care facilities: The effect of organizational cultures on staff attitudes, knowledge, practices and infection acquisition rates. American Journal of Infection. 2015; 43: 935-939.

25. Strauss A, Corbin J. Basics of qualitative research: Grounded theory procedures and techniques. Newbury Park, CA: Sage. 1990.

(C) 2018 McGhie-Anderson RL. This article is distributed under the terms of the Creative Commons Attribution 4.0 International License 